\title{
Probabilistically Bounded Staleness for Practical Partial Quorums
}

\author{
Peter Bailis \\ Shivaram Venkataraman \\ Joseph M. Hellerstein \\ Michael Franklin \\ Ion Stoica
}

Electrical Engineering and Computer Sciences University of California at Berkeley

\author{
Technical Report No. UCB/EECS-2012-4 \\ http://www.eecs.berkeley.edu/Pubs/TechRpts/2012/EECS-2012-4.html \\ January 3, 2012
}


Copyright (C) 2012, by the author(s).

All rights reserved.

Permission to make digital or hard copies of all or part of this work for personal or classroom use is granted without fee provided that copies are not made or distributed for profit or commercial advantage and that copies bear this notice and the full citation on the first page. To copy otherwise, to republish, to post on servers or to redistribute to lists, requires prior specific permission. 


\title{
Probabilistically Bounded Staleness for Practical Partial Quorums
}

\author{
Peter Bailis, Shivaram Venkataraman, Michael J. Franklin, Joseph M. Hellerstein, Ion Stoica \\ University of California, Berkeley \\ \{pbailis, shivaram, franklin, hellerstein, istoica\}@cs.berkeley.edu
}

All good ideas arrive by chance.-Max Ernst

\begin{abstract}
Modern storage systems employing quorum replication are often configured to use partial, non-strict quorums. These systems wait only for a subset of their replicas to respond to a request before returning an answer, without guaranteeing that read and write replica sets intersect. While these partial quorum mechanisms provide only basic eventual consistency guarantees, with no limit to the recency of data returned, these configurations are frequently "good enough" for practitioners given their latency benefits. In this work, we discuss why partial quorums are often acceptable in practice by analyzing the staleness of data they return. Extending prior work on strongly consistent probabilistic quorums and using models of Dynamo-style anti-entropy processes, we introduce Probabilistically Bounded Staleness (PBS) consistency, which provides expected bounds on staleness with respect to both versions and wall clock time. We derive a closed-form solution for versioned staleness and model real-time staleness for representative Dynamo-style systems under internet-scale production workloads. We quantitatively demonstrate why, in practice, eventually consistent systems employing partial quorums often serve consistent data.
\end{abstract}

\section{INTRODUCTION}

Modern distributed storage systems need to be scalable, highly available, and fast. These systems typically replicate data across different machines and often across datacenters for two reasons: first, to provide high availability when components fail and, second, to provide improved performance by serving requests from multiple replicas. In order to provide predictably low read and write latency, systems often eschew protocols guaranteeing consistency of reads ${ }^{1}$ and instead opt for eventually consistent protocols $[4,6,21$, $23,38,39,56]$. However, weak eventually consistent systems make no guarantees on the staleness (recency in terms of versions written) of data items returned except that the system will "eventually" return the most recent version in the absence of new writes [62].

\footnotetext{
${ }^{1}$ Note that this distributed replica consistency is different from transactional consistency provided by ACID semantics [51, 59].
}

Distributed quorums can be used to ensure strong consistency across multiple replicas of a data item by overlapping read and write replica sets. However, waiting for responses from the potentially large resulting quorum sizes increases operation latency, an important consideration for service operators [57]. For example, at Amazon, $100 \mathrm{~ms}$ of additional latency resulted in a 1\% drop in sales [44], while $500 \mathrm{~ms}$ of additional latency in Google's search resulted in a corresponding $20 \%$ decrease in traffic [45]. At scale, these decreases correspond to large amounts of lost revenue.

Employing partial or non-strict quorums lowers operation latency in quorum replication. With partial quorums, sets of replicas written to and read from are not guaranteed to overlap: given $N$ replicas and read and write quorum sizes $R$ and $W$, partial quorums imply $R+W \leq N$. Modern quorum-based data systems such as Dynamo [21] and its open source descendants Apache Cassandra [41], Basho Riak [3], and Project Voldemort [24] offer a choice between these two modes of quorum replication: overlapping quorums, providing strong consistency, and partial quorums, providing eventual consistency.

Operators frequently employ partial quorums $[1,4,23,38,56$, 64] despite the weak guarantees provided by eventual consistencya controversial decision $[32,47,58,59]$. These partial quorums are often acceptable for operators due to their latency benefits, which are especially important as latencies grow (e.g., a wide-area network scenario) $[6,23,32,33]$. Many programs are carefully designed to handle staleness through design patterns such as compensation (e.g., memories, guesses, and apologies) [33] and associative and commutative operations (e.g., timelines, logs, and notifications) [12]. In general, however, unbounded staleness poses significant challenges and is undesirable in practice. The proliferation of partial quorum configurations suggests that many applications can tolerate occasional cases of staleness and that stale data tends to be "fresh enough" in most cases.

While common practice suggests that weak eventual consistency is a viable solution for many operators, to date, this observation has been mostly anecdotal. In this work, we quantify the degree to which eventual consistency is both eventual and consistent and explain why. Under worst-case conditions, eventual consistency results in an unbounded degree of data staleness, but, as we will show, the average case is often different. Eventually consistent data stores cannot promise immediate and perfect consistency but, for varying degrees of certainty, can offer staleness bounds with respect to time ("how eventual") and version history ("how consistent"). There is little prior work describing how to make these consistency and staleness predictions under practical conditions. The current state of the art requires that users make rough guesses or perform online profiling to determine the consistency provided by their data stores.

To do better, we need to know when and why eventually consis- 
tent systems return stale data and how to quantify the staleness of the data they return. In this work, we answer these questions in the context of quorum replicated data stores by expanding theoretical research on probabilistic quorums $[50,52]$ to account for multiversion staleness, anti-entropy [22], and quorum dissemination protocols as used in today's systems. More precisely, we present algorithms and models for predicting the staleness of partial quorums, called Probabilistically Bounded Staleness (PBS) for partial quorums. There are two common axes for measuring staleness in the literature: versions [40, 67] and wall clock time [65, 66]. PBS can be used to analyze both measures, describing the probability of reading a version $t$ seconds after it is written ( $t$-visibility, or "how eventual is eventual consistency?"), of reading one of the last $k$ versions of a data item ( $k$-staleness, or "how consistent is eventual consistency?"), and of experiencing a combination of the two $(\langle k, t\rangle$-staleness). PBS does not propose new mechanisms to enforce deterministic staleness bounds [40, 55, 65, 66, 67]; instead, our goal is to provide a lens for analyzing and improving the behavior of existing, widely-deployed systems.

We provide closed-form solutions for PBS $k$-staleness and use Monte Carlo methods to explore the trade-off between latency and $t$-visibility. We present a detailed study of Dynamo-style PBS $t$ visibility using production latency distributions to explain why realworld operators frequently choose to employ partial quorums. We show how relatively long-tailed one-way write latency distributions affect the time required for a high probability of consistent reads. We quantitatively demonstrate how moving from spinning disks to solid-state drives has a large impact on staleness (e.g., $1.85 \mathrm{~ms}$ versus $45.5 \mathrm{~ms}$ wait time required for a $99.9 \%$ probability of consistent reads). We also make quantitative observations regarding the latency-consistency trade-offs offered by partial quorums. For example, under another production workload, we observe an $81.1 \%$ latency improvement at the 99.9 th percentile (230 to $43.3 \mathrm{~ms}$ ) for a $202 \mathrm{~ms}$ window of inconsistency with $99.9 \%$ confidence. While the benefit of these trade-offs is application-specific, our analysis demonstrates many of the performance benefits that lead many operators to choose weak consistency.

We make the following contributions in this paper:

- We develop the theory of Probabilistically Bounded Staleness (PBS) for partial quorums. PBS describes the probability of staleness across both versions ( $k$-staleness) and time $(t$-visibility) as well as the probability of monotonic reads consistency.

- We provide a closed-form analysis of $k$-staleness demonstrating how the probability of receiving data $k$ versions stale is exponential in $k$. As a corollary, $k$-staleness tolerance also exponentially lowers quorum system load.

- We provide a model for $t$-visibility in Dynamo-style partial quorum systems, WARS, showing how staleness is dependent on message reordering due to latency. We evaluate the $t$ visibility of Dynamo-style systems using a combination of synthetic and production latency models.

\section{BACKGROUND}

In this section, we provide background regarding quorum systems both in theoretical academic literature and in practice. We begin by introducing prior work on traditional and probabilistic quorum systems. We next discuss Dynamo-style quorums, currently the most widely deployed protocol for storage systems employing quorum replication. Finally, we survey reports of practitioner usage of partial quorums for three Dynamo-style data systems.

\subsection{Quorum Foundations: Theory}

Quorum systems have long been proposed as a replication strategy for distributed data [26]. Under quorum replication, a data storage system writes a data item by sending it to a set of replicas, called a write quorum. To serve reads, the data system fetches the data from a possibly different set of replicas, called a read quorum. For reads, the storage system compares the set of values returned by the replicas, and, given a total ordering over versions of the data item $^{2}$, can return the most recent value (or all values received, if desired). For each operation, read and write quorums are chosen from a set of sets of replicas, known as a quorum system, with one system per data item. There are many ways to configure quorum systems, but one simple solution is to use read and write quorums of fixed sizes, which we will denote $R$ and $W$, respectively, for a set of nodes of size $N$. To reiterate, a quorum replicated data system uses one quorum system per data item. Across data items, the quorum systems need not be identical

Informally, a strict quorum system is a quorum system with the property that any two quorums (sets) in the quorum system overlap (have non-empty intersection). This ensures consistency. The minimum sized quorum defines the system's fault tolerance, or availability. A simple example of a strict quorum system is the majority quorum system, in which each quorum is of size $\left\lceil\frac{N}{2}\right\rceil$. However, the theory literature contains many alternative quorum system designs providing varying asymptotic properties of capacity, scalability, and fault tolerance, from tree-quorums [9] to grid-quorums [53] and highly-available hybrids [8]. Jiménez-Peris et. al provide an overview of traditional, strict quorum systems [37].

Partial quorum systems are natural extensions of strict quorum systems: at least two quorums in a partial quorum system do not overlap. There are two relevant variants of partial quorum systems in the literature: probabilistic quorum systems and k-quorums.

Probabilistic quorum systems provide probabilistic guarantees of quorum intersection. By scaling the number of replicas, we can achieve an arbitrarily high probability of consistency [50]. Intuitively, this is a consequence of the Birthday Paradox: as the number of replicas increases, the probability of non-intersection between any two quorums decreases. To the best of our knowledge, probabilistic quorums have only been used to study the probability of strong consistency and have not been used to study bounded staleness, particularly in the presence of anti-entropy [22]. Merideth and Reiter provide an overview of these systems [52].

As an example of a probabilistic quorum system, given $N$ replicas and randomly chosen read and write quorums of sizes $R$ and $W$, we can calculate the probability that the read quorum does not contain the last written version. This probability of inconsistency is the number of quorums of size $R$ composed of nodes that were not written to in the write quorum divided by the number of possible quorums of size $R$ :

$$
p_{s}=\frac{\left(\begin{array}{c}
N-W \\
R
\end{array}\right)}{\left(\begin{array}{c}
N \\
R
\end{array}\right)}
$$

The probability of inconsistency is high except for large $N$. With $N=100, R=W=30, p_{s}=1.88 \times 10^{-6}$ [10]. However, with $N=3, R=W=1, p_{s}=. \overline{6}$. The asymptotics of these systems are excellent—but only asymptotically.

$k$-quorum systems provide deterministic guarantees that a partial quorum system will return values that are within $k$ versions of the most recent write [10]. In the single writer scenario, a round-robin

\footnotetext{
${ }^{2}$ This total ordering can be achieved using globally synchronized clocks [46] or using a causal ordering provided by mechanisms such as vector clocks [42] with commutative merge functions [47]
} 
write scheduling scheme where each write is sent to $\frac{N}{k}$ replicas ensures that any replica is no more than $k$ versions out-of-date. However, with multiple writers, the global ordering properties that the single-writer was able to control are lost, and the best known algorithm for the pathological case results in a lower bound of $(2 N-1)(k-1)+N$ versions staleness [11].

This prior work makes two important assumptions. First, it typically models quorum sizes as fixed, where the set of nodes with a version does not grow over time. Prior work explored "dynamic systems" in terms of quorum membership churn [7], network-aware quorum placement [25, 29], and network partitions [34] but did not model write propagation. Second, it frequently assumes Byzantine failure. We revisit these assumptions in the next section.

\subsection{Quorum Foundations: Practice}

In practice, many distributed data management systems use quorums as a replication mechanism. Amazon's Dynamo [21] is the progenitor of a class of eventually-consistent key-value stores that includes Apache Cassandra ${ }^{3}, \mathrm{Riak}^{4}$, and Voldemort ${ }^{5}$. All use the same variant of quorum-style replication, and we are not aware of any significantly different, widely adopted data systems using quorum replication. However, with some work, we believe that other styles of replication can adopt our methodology. We describe keyvalue stores here, but any replicated data store can use quorums, including full RDBMS systems.

Dynamo-style quorum systems employ one quorum system per key, typically maintaining the mapping of keys to quorum systems using a consistent-hashing scheme or a centralized membership protocol. Each node stores multiple keys. As shown in Figure 1, client read and write requests are sent to a node in the system cluster, which forwards the request to all nodes assigned to that key as replicas. The coordinating node considers an operation complete when it has received responses from a pre-defined number of replicas. Accordingly, without message loss, all replicas eventually receive all writes. This means that the write and read quorums chosen for a request depend on which nodes respond to the request first. In Dynamo terminology, the quorum size, or replication factor, is defined as $N$, the number of replica responses required for a successful read is defined as $R$, and the number of replica acknowledgments required for a successful write is defined as $W$. Dynamostyle systems are guaranteed to be consistent when $R+W>N$. Setting $W>\lceil N / 2\rceil$ ensures that a majority of replicas will will receive a write in the presence of multiple concurrent write requests.

There are significant differences between quorum theory and data systems used in practice. First, replication factors for distributed data systems are relatively low. Typical replication factors are between one and three $[4,23,30]$. Second, (in the absence of failure), in Dynamo-style partial quorums, the write quorum size increases even after the operation returns, growing via anti-entropy [22]. Moreover, requests are sent to all replicas, however only the first $R$ responses are considered. As a matter of nomenclature (and to disambiguate against "dynamic" quorum membership protocols), we will refer to these systems as expanding partial quorum systems. We discuss additional anti-entropy in Section 4.2. Third, as in much of the practical literature, practitioners largely focus on fail-stop failure instead of Byzantine failure [17]. Following standard practice, we do not consider Byzantine failure.

\footnotetext{
${ }^{3}$ Apache Cassandra. cassandra . apache .org

${ }^{4}$ Basho Riak. www . basho.org

${ }^{5}$ Project Voldemort. www.project-voldemort.com
}

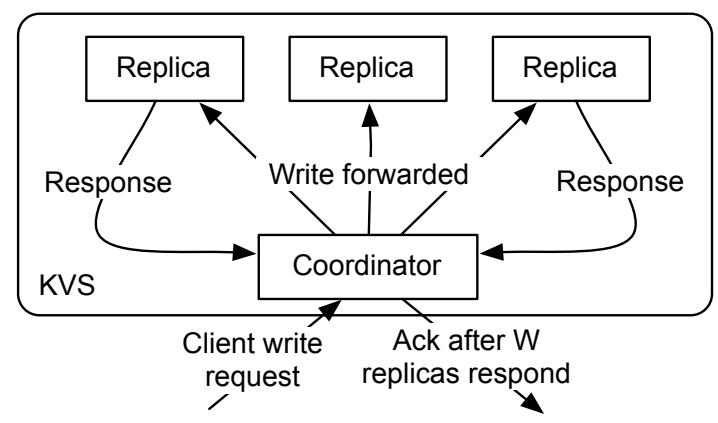

Figure 1: Diagram of control flow for client write to Dynamostyle quorum. Here, $N=3, W=2$. The client write is handled by a coordinator node and sent to all replicas. The write succeeds when $W$ replicas respond. Note that the coordinator is possibly a replica as well, invoking a local write.

\subsection{Typical Quorum Configurations}

For improved latency, operators often set $R+W \leq N$. Here, we survey quorum configurations according to practitioner accounts. Many operators appear to use partial quorum configurations, frequently citing performance benefits and high availability.

Cassandra defaults to $N=3, R=W=1$ [4]. The Apache Cassandra 1.0 documentation claims that "a majority of users do writes at consistency level $[W=1]$ ", while the Cassandra Query Language defaults to $R=W=1$ as well [1]. Production Cassandra users report using $R=W=1$ in the "general case" because it provides "maximum performance" [64], which appears to be a commonly held belief $[38,56]$. Cassandra has a "minor" patch [2] for session guarantees [61] that is not currently used [16]; according to our discussions with developers, this is due to lack of interest.

Riak defaults to $N=3, R=W=2[14,15]$. Users suggest using $R=W=1, N=2$ for "low value" data (and strict quorum variants for "web," "mission critical," and "financial" data) [39, 48].

Finally, Voldemort does not provide sample configurations, but Voldemort's authors (and operators) at LinkedIn [23] often choose $N=c, R=W=\lceil c / 2\rceil$ for odd $c$. For applications requiring "very low latency and high availability," LinkedIn deploys Voldemort with $N=3, R=W=1$. For other applications, LinkedIn deployments Voldemort with $N=2, R=W=1$, providing "some consistency," particularly when $N=3$ replication is not required. Additionally, Voldemort supports a concept of preferred reads and writes, meaning it will block until either the preferred number of replicas respond or a timeout occurs, at which point the request succeeds. In the low latency case, preferred reads is either two or is disabled. In the $N=2$ case, preferred reads and preferred writes are set to two. Voldemort also differs from Dynamo in that it sends read requests to $R$ of $N$ replicas (not $N$ of $N$ ) [24]; this decreases load per replica and network traffic at the expense of read latency and potential availability. Provided the per-request staleness probabilities are independent, this does not affect staleness: even when sending reads to $N$ replicas, coordinators only wait for $R$ responses.

\section{PROBABILISTICALLY BOUNDED STALENESS}

In this section, we introduce Probabilistically Bounded Staleness, which describes the consistency provided by existing eventually consistent data stores. We introduce the notions of PBS $k$-staleness, which stochastically bounds the staleness of versions returned by read quorums, PBS $t$-visibility, which stochastically bounds the time before a committed version appears to readers, and PBS $\langle k, t\rangle$-staleness, a combination of the two prior models. 


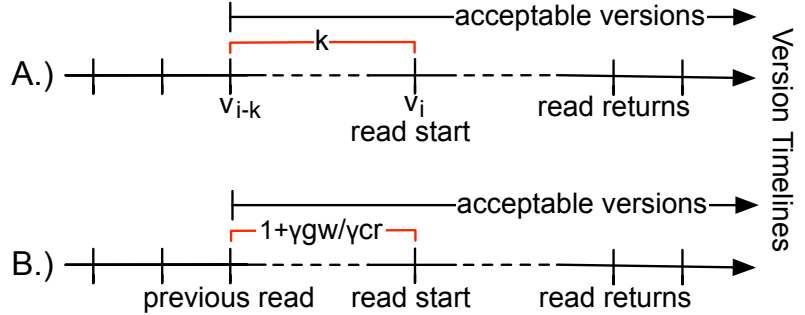

Figure 2: Versions returnable by read operations under PBS $k$-staleness (A) and PBS monotonic reads (B). In $k$-staleness, the read operation will return a version no later than $k$ versions older than the last committed value when it started; later versions may be committed during the read and may also be returned. In monotonic reads consistency, acceptable staleness depends on the number of versions committed since the client's last read.

We first introduce $k$-staleness because it is self-contained, with a simple closed-form solution. In comparison, $t$-visibility is more difficult, involving several additional variables. Accordingly, this section proceeds in order of increasing difficulty, and the remainder of the paper largely addresses the complexities of $t$-visibility.

Practical concerns guide the following theoretical contributions. We begin by considering a model without quorum expansion or other anti-entropy. For the purposes of a running example, as in Equation 1, we assume that $W(R)$ of $N$ replicas are randomly selected for each write (read) operation. Similarly, we consider fixed $W, R$ and $N$ across multiple operations. Next, we expand our model to consider write propagation and time-varying $W$ sizes in expanding partial quorums. In this section, we discuss anti-entropy in general, however we model Dynamo-style quorums in Section 4. We discuss further refinements to our assumptions in Section 6 .

\subsection{PBS $k$-staleness}

Probabilistic quorums allow us to determine the probability of returning the most recent value written to the database, but do not describe what happens when the most recent value is not returned. Here, we determine the probability of returning a value within a bounded number of versions. In the following formulation, we consider traditional, non-expanding write quorums (no anti-entropy):

Definition 1. A quorum system obeys $P B S k$-staleness consistency if, with probability $1-p_{s k}$, at least one value in any read quorum will have been committed within $k$ versions of the latest committed version when the read begins.

Versions whose writes that are not yet committed (in-flight) may be returned by a read (see Figure 2A). The $k$-quorum literature defines these as $k$-regular semantics [10].

The probability of returning a version of a key within the last $k$ versions committed is equivalent to intersecting one of $k$ independent write quorums. Given the probability of a single quorum intersection $p$, the probability of intersecting one of the last $k$ independent quorums is $p^{k}$. In our example quorum system, the probability of non-intersection is Equation 1 exponentiated by $k$ :

$$
p_{s k}=\left(\frac{\left(\begin{array}{c}
N-W \\
R
\end{array}\right)}{\left(\begin{array}{c}
N \\
R
\end{array}\right)}\right)^{k}
$$

When $N=3, R=W=1$, this means that the probability of returning a version within 2 versions is.$\overline{5}$, within 3 versions.$\overline{703}$, and within 5 versions $>.868$, and 10 versions $>.98$. When $N=3, R=1, W=2$ (equivalently, $R=2, W=1$ ), these probabilities increase: $k=1 \rightarrow . \overline{6}, k=2 \rightarrow \overline{8}$, and $k=5 \rightarrow>.995$.
This closed form solution holds for quorums that do not change size over time. For expanding partial quorum systems, this solution is an upper bound on the probability of staleness. We discuss the load improvements offered by PBS $k$-staleness as discussed in the quorum system literature in Appendix A.

\subsection{PBS Monotonic Reads}

PBS $k$-staleness can be used to predict whether a client will ever older staler data than it has already read, a well-known session guarantee called monotonic reads consistency [61]. This is particularly useful when clients do not need to see the most recent version of a data item but still require a notion of "forward progress" through versions, as in streaming changelogs or timelines.

Definition 2. A quorum system obeys $P B S$ monotonic reads consistency if, with probability at least $1-p_{s M R}$, at least one value in any read quorum returned to a client is the same version or a newer version than the last version that the client previously read.

To guarantee that a client sees monotonically increasing versions, it can continue to contact the same replica [62] (provided the "sticky" replica does not fail). However, this is insufficient for strict monotonic reads (where the client reads strictly newer data if it exists in the system). Definition 2 can be adapted to accommodate strict monotonic reads by requiring that, if a more recent data version is available, it is returned.

PBS monotonic reads consistency is a special case of PBS $k$ staleness (see Figure 2B), where $k$ is determined by a client's rate of reads from a data item $\left(\gamma_{c r}\right)$ and the global, system-wide rate of writes to the same data item $\left(\gamma_{g w}\right)$. If we know these rates exactly, the number of versions between client reads is $\frac{\gamma_{g w}}{\gamma_{c r}}$, as shown in Figure $2 \mathrm{~B}$. We can calculate the probability of probabilistic monotonic reads as a special case of $k$-staleness where $k=1+\frac{\gamma_{g w}}{\gamma_{c r}}$. For example, extending our running example from Equation 2:

$$
p_{s M R}=\left(\frac{\left(\begin{array}{c}
N-W \\
R
\end{array}\right)}{\left(\begin{array}{c}
N \\
R
\end{array}\right)}\right)^{1+\gamma_{g w} / \gamma_{c r}}
$$

For strict monotonic reads, where we cannot read the version we have previously read (assuming there are newer versions in the database), we exponentiate with $k=\frac{\gamma_{g w}}{\gamma_{c r}}$.

In practice, we may not know these exact rates, but, by measuring their distribution, we can calculate an expected value. By performing appropriate admission control, operators can control these rates to achieve monotonic reads consistency with high probability.

\subsection{PBS $t$-visibility}

Until now, we have considered only quorums that do not grow over time. However, as we discussed in Section 2.2, real-world quorum systems expand by asynchronously propagating writes to quorum system members over time. This process is commonly known as anti-entropy [22]. For generality, in this section, we will discuss generic anti-entropy. However, we explicitly model the Dynamo-style anti-entropy mechanisms in Section 4.

PBS $t$-visibility models the probability of inconsistency for expanding quorums. Intuitively, PBS $t$-visibility captures the possibility that a reader will observe a write $t$ seconds after it commits. Recall that we consider in-flight writes-which are more recent than the last committed version-as non-stale.

Definition 3. A quorum system obeys $P B S t$-visibility consistency if, with probability $1-p_{s t}$, any read quorum started at least $t$ units of time after the last version committed returns at least one value that is at least as recent as the last committed version. 
Overwriting data items effectively resets $t$-visibility; $t$-visibility time is bounded by the time between writes. Intuitively, if two writes to a key are spaced $m$ milliseconds apart, then the $t$-visibility of the first write for $t>m$ milliseconds is undefined; after $m$ milliseconds, there will be a newer version.

We denote the cumulative density function describing the number of replicas $\mathcal{W}_{r}$ that have received a particular version $v$ (or a version newer than $v$ ) $t$ seconds after $v$ commits as $P_{w}\left(\mathcal{W}_{r}, t\right)$.

By definition, for expanding quorums, $\forall c \in[0, W], P_{w}(c, 0)=$ 1 ; at commit time, $W$ replicas will have received the value with certainty. We can model the probability of PBS $t$-visibility for given $t$ by summing the conditional probabilities of each possible $\mathcal{W}_{r}$ :

$$
p_{s t}=\frac{\left(\begin{array}{c}
N-W \\
N
\end{array}\right)}{\left(\begin{array}{l}
N \\
R
\end{array}\right)}+\sum_{c \in(W, N]} \frac{\left(\begin{array}{c}
N-c \\
N
\end{array}\right)}{\left(\begin{array}{l}
N \\
R
\end{array}\right)} \cdot\left[P_{w}(c+1, t)-P_{w}(c, t)\right]
$$

However, the above equation assumes reads occur instantaneously and writes commit immediately after $W$ replicas have the version (i.e., there is no delay acknowledging the write to the coordinating node). In the real world, writes need to be acknowledged and read requests take time to arrive at remote replicas, increasing $t$. Accordingly, Equation 4 is a conservative upper bound on $p_{s t}$.

In practice, $P_{w}$ depends on the anti-entropy mechanisms in use and the expected latency of operations and can be approximated (Section 4) or measured online. For this reason, the load of a PBS $t$ visible quorum system depends on write propagation and is difficult to analytically determine for general-purpose expanding quorums. Additionally, one can model both transient and permanent failures by increasing the tail probabilities of $P_{w}$ (Section 6).

\subsection{PBS $\langle k, t\rangle$-staleness}

We can combine the previous models to combine both versioned and real-time staleness metrics to determine the probability that a read will return a value no older than $k$ versions stale if the last write committed no sooner than $t$ seconds ago:

Definition 4. A quorum system obeys $P B S\langle k, t\rangle$-staleness consistency if, with probability $1-p_{s k t}$, at least one value in any read quorum will be within $k$ versions of the latest committed version when the read begins, provided the read begins $t$ units of time after the previous $k$ versions commit.

The definition of $p_{s k t}$ follows from the prior definitions:

$p_{s k t}=\left(\frac{\left(\begin{array}{c}N-W \\ R\end{array}\right)}{\left(\begin{array}{l}N \\ R\end{array}\right)}+\sum_{c \in[W, N)} \frac{\left(\begin{array}{c}N-c \\ R\end{array}\right)}{\left(\begin{array}{l}N \\ R\end{array}\right)} \cdot\left[P_{w}(c+1, t)-P_{w}(c, t)\right]\right)^{k}$

In this equation, in addition to (again) assuming instantaneous reads, we also assume the pathological case where the last $k$ writes all occurred at the same time. If we can determine the time since commit for the last $k$ writes, we can improve this bound by considering each quorum's $p_{s k t}$ separately (individual $t$ ). However, predicting (and enforcing) write arrival rates is challenging and may introduce inaccuracy, so this equation is a conservative upper bound on $p_{s k t}$.

Note that the prior definitions of consistency are encapsulated by PBS $\langle k, t\rangle$-staleness consistency. Probabilistic $k$-quorum consistency is simply PBS $\langle k, 0\rangle$-staleness consistency, PBS monotonic reads consistency is $\left\langle 1+\frac{\gamma_{g w}}{\gamma_{c r}}, 0\right\rangle$-staleness consistency, and PBS $t$-visibility is $\langle 1, t\rangle$-staleness consistency.

In practice, we believe it is easier to reason about staleness of versions or staleness in terms of real time but not both together. Accordingly, having derived a closed-form model for $k$-staleness, in the remainder of this paper, we focus mainly on deriving more specific models for $t$-visibility.

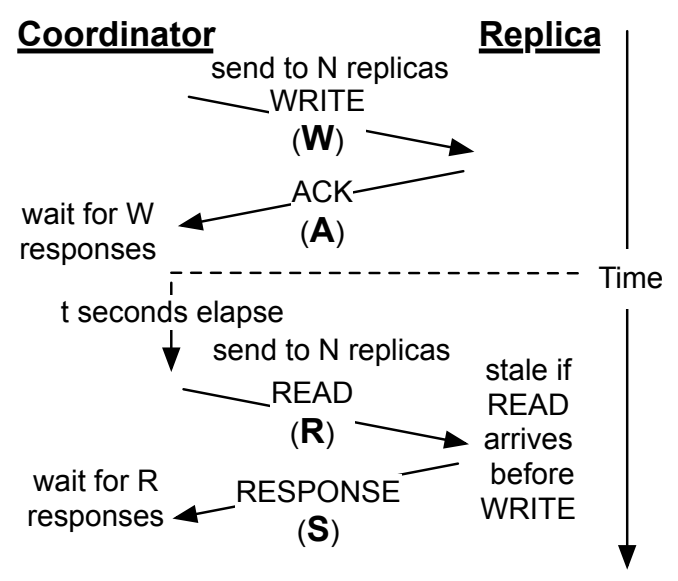

Figure 3: The WARS model for message ordering in Dynamo describes the message flow and latencies between a coordinator and a single replica for a write followed by a read $t$ seconds after commit. In an $N$ replica system, this message flow occurs $N$ times, once for each of the $N$ replicas. The read and write may be handled by different coordinators.

\section{DYNAMO-STYLE $T$-VISIBILITY}

We have a closed-form analytical model for $k$-staleness, but $t$ visibility is dependent on both the distributed quorum replication algorithm and the anti-entropy processes employed by a data storage system. In this section, we discuss PBS $t$-visibility in the context of Dynamo-style data storage systems. We describe how to model the probability of staleness in these systems and how to asynchronously detect staleness.

\subsection{Inconsistency in Dynamo: WARS Model}

Dynamo-style quorum systems are inconsistent as a result of read and write message reordering, a product of message delays. Reads and writes are sent to all quorum members, so the staleness under normal operation results only when all of the first $R$ responses to a read request arrived at their respective replicas before the last committed write request. To illustrate this phenomenon, we introduce a model of message latency in Dynamo operation which, for convenience, we will call WARS.

In Figure 3, we illustrate WARS using a space-time diagram for messages between a coordinator and a single replica for a write followed by a read $t$ seconds after the write commits. This $t$ corresponds to the $t$ in PBS $t$-visibility.

For a write, the coordinator sends $N$ messages, one to each replica. The message from coordinator to replica containing the version to be written is delayed by a value chosen from distribution W. The coordinator waits for $W$ responses from the replicas before it can consider the version committed. Each response acknowledging the write is delayed by a value chosen from the distribution A.

For a read, the coordinator sends $N$ messages, one to each replica. The message from coordinator to replica containing the read request is delayed by a value chosen from distribution $R$. The coordinator waits for $R$ responses from the replicas before returning the most recent value it receives. The read response from each replica is delayed by a value chosen from the distribution $\mathrm{S}$.

The read coordinator will return stale data if the first $R$ responses received reached their respective replicas before the replicas received the latest version (delayed by W). When $R+W>N$, this is impossible. However, under partial quorums, this depends on the latency distributions. If we denote the commit time as $w_{t}$, a single replica's response is stale if $r^{\prime}+w_{t}+t<w^{\prime}$ for $r^{\prime}$ chosen 
from $\mathrm{R}$ and $w^{\prime}$ chosen from $\mathrm{W}$. Writes have time to propagate to additional replicas both while the coordinator waits for all required acknowledgments (A) and as read requests are subsequently sent (R). Similarly, read responses are further delayed in transit (S) back to the read coordinator, inducing further possibility of reordering. Qualitatively, longer write tails and faster reads increase the chance of staleness due to the possibility of reordering.

WARS considers the effect of message sending, delays, and reception, but this represents a daunting analytical formulation. The commit time represents an order statistic of $W$ and $N$ dependent on both $\mathrm{W}$ and A. Furthermore, the probability that the $i$ th returned read message observes reordering is another order statistic of $R$ and $N$ dependent on W,A,R, and S. Moreover, across responses, the probabilities are dependent. These intertwined dependencies make calculating the probability of staleness rather difficult. Dynamo is straightforward to reason about and program but is difficult to analyze in a simple closed form, which eludes us. As we discuss in Section 5.1, we explore WARS using Monte Carlo methods, which are straightforward to understand and implement.

\subsection{WARS Scope}

Proxying operations. Depending on which coordinator a client contacts, some reads and writes may be served locally. In this case, subject to local query processing delays, a read or write to $R$ or $W$ nodes behaves like a read or write to $R-1$ or $W-1$ nodes, respectively. Although we do not do so, WARS can be adopted to handle local reads and writes. Determining whether requests will be proxied (and, if not, which replicas serve which requests) is data store and deployment-specific. Dynamo forwards write requests to a designated coordinator solely for the purpose of establishing a version ordering [21, Section 6.4] (easily achievable through other mechanisms [36]). Dynamo's authors observed a latency improvement by proxying all operations and having clients act as coordinatorsVoldemort adopts this architecture [60].

Client-side delays. Many end-users will incur additional time between their respective reads and writes due to latency required to contact the service. Individuals making requests to web services through their browsers will likely space sequential requests by tens or hundreds of milliseconds due to client-to-server latency. Although we do not consider this delay here, it is important to remember for practical scenarios because the delay between reads and writes $(t)$ may be large.

Additional anti-entropy. As we discussed in Section 2.2, antientropy decreases the probability of staleness by further propagating versions between members. Dynamo-style systems also support additional anti-entropy processes [51]. One common process is called read repair: when a read coordinator receives multiple versions of a data item from different replicas in response to a read request, it will attempt to (asynchronously) update the out-of-date replicas with the most recent version [21, Section 5]. Read repair acts like an additional write for every read, except old values are re-written. Additionally, Dynamo used Merkle trees to summarize and exchange data contents between replicas [21, Section 4.7]. However, not all Dynamo-style data stores actively employ similar gossip-based anti-entropy. For example, Cassandra only uses Merkle tree anti-entropy when it is manually requested (e.g., nodetool repair), choosing to rely primarily on quorum expansion and read repair [5].

Both of these processes are rate-dependent: read repair effects depend on the rate of reads, and Merkle tree exchange effects (and, more generally, most anti-entropy processes) depend on the rate of exchange. A conservative assumption for read repair and Merkle tree exchange is that they never occur; for example, without ad- ditional modifications to the Dynamo protocol, assuming a read repair rate is equivalent to assuming a rate of reads from each key in the system. WARS only captures expanding quorum behavior but is read rate independent and makes conservative assumptions about the write rate. If multiple writes overlap (that is, have overlapping periods where they are in-flight but are not committed) the probability of inconsistency decreases. Intuitively, this is because overlapping writes result in an increased chance that a client reads as-yet-uncommitted data. Versions may be fresher than predicted.

\subsection{Asynchronous Staleness Detection}

Even if a system provides a low probability of inconsistency, applications may need to be notified when data returned is inconsistent or staler than expected. Here, as a side note, we discuss how the Dynamo protocol is naturally equipped for staleness detection. The following discussion is couched in the terms of PBS $t$-visibility but is easily extended to PBS $k$-staleness and $\langle k, t\rangle$-staleness.

Knowing whether a response is stale at read time requires strong consistency. By checking all possible values in the domain against a hypothetical staleness detector, we could determine the consistent value to return. While we cannot do so synchronously, we can determine staleness asynchronously. Asynchronous staleness detection allows speculative execution [63] if a program contains appropriate compensation logic.

We first consider a staleness detector providing false positives. Recall that, in a Dynamo-style system, we wait for $R$ of $N$ replies before returning a value. The remaining $N-R$ replicas will still reply to the read coordinator. Instead of dropping these messages, the coordinator can compare them to the version it returned. If there is a mismatch, then either the coordinator returned stale data, there are in-flight writes in the system, or additional versions committed after the read. The latter two cases, relating to data committed after the response was initiated, lead to false positives. In these cases, the read did not return "stale" data even though there were newer but uncommitted versions in the system. Notifying clients about newer but uncommitted versions of a data item is not necessarily bad but may be unnecessary and violates our staleness semantics. This detector does not require modifications to the Dynamo protocol and is similar to the read-repair process.

To eliminate these uncommitted-but-newer false positives, we need to determine the total, system-wide commit ordering of writes. Recall that replicas are unaware of the commit time for each version; commits occur after $W$ replicas respond, and the timestamps stored by replicas are not updated after commit. Establishing a total ordering is a well-known distributed systems problem that could be accomplished in Dynamo using a centralized service [36] or using distributed consensus [43]. This requires modifications to the Dynamo protocol but is feasible.

\section{EVALUATING DYNAMO $T$-VISIBILITY}

As discussed in Section 3.3, PBS $t$-visibility depends on the propagation of reads and writes throughout a system. We introduced the WARS model as a means of reasoning about inconsistency in Dynamo-style quorum systems, but quantitative metrics such as staleness observed in practice depend on each of WARS's latency distributions. In this section, we perform an analysis of Dynamo-style $t$-visibility to better understand how frequently "eventually consistent" means "consistent" and, more importantly, why.

PBS $k$-staleness is easily captured in closed form (Section 3.1). It does not depend on write latency or any environmental variables. Indeed, in practice, without expanding quorums or anti-entropy, we observe that our derived equations hold true experimentally.

$t$-visibility depends on anti-entropy, which is substantially more 
complicated. In this section, we focus on deriving experimental expectations for PBS $t$-visibility. While we could improve the staleness results by considering additional anti-entropy processes (Section 4.2), we make the bare minimum of assumptions required by the WARS model. Conservative analysis decreases the number of experimental variables (supported by empirical observations from practitioners) and increases the applicability of our results.

\subsection{Monte Carlo Simulation}

In light of the complicated analytical formulation discussed in Section 4.1, we implemented WARS in an event-driven simulator for use in Monte Carlo methods. Calculating $t$-visibility for a given value of $t$ is straightforward: draw $N$ samples from $\mathrm{W}, \mathrm{A}, \mathrm{R}$, and $\mathrm{S}$ at time $t$ (denote index $i$ as $[i]$ ), compute $w_{t}$, the $W$ th smallest value of the respective $\mathrm{W}+\mathrm{A}$ values, and check whether the first $R$ samples of $\mathrm{R}$, ordered by $\mathrm{R}[i]+\mathrm{S}[i]$ obey $w_{t}+\mathrm{R}[i]+t \leq \mathrm{W}[i]$. This requires only a few lines of code. Extending this formulation to analyze $\langle k, t\rangle$-staleness given a distribution of write arrival times requires accounting for multiple writes across time but is not difficult.

\subsection{Model Validation}

To validate WARS (and our subsequent analyses), we compared our predicted $t$-visibility and latency with measured values observed in a commercially available, open source Dynamo-style key-value store. We modified Cassandra to provide latency measurements, disabled read repair (as it is external to WARS), and, for reads, only considered the first $R$ responses (often, more than $R$ messages would arrive by the processing stage, decreasing staleness). We ran Cassandra on a cluster of four nodes with $2.2 \mathrm{GHz}$ AMD Opteron 2214 dual-core SMT processors with $4 \mathrm{~GB}$ of $667 \mathrm{MHz}$ DDR2 memory serving in-memory data. To measure staleness, we inserted monotonically increasing versions of a key while concurrent processes read values. Solely for the purpose of validation across several conditions, we injected additional latency into Cassandra's messaging.

Our observations matched the WARS predictions. We injected each combination of $\mathrm{W}=\lambda \in\{0.05,0.1,0.2\}$ (respective means $20 \mathrm{~ms}, 10 \mathrm{~ms}$ and $5 \mathrm{~ms}$ ) and $\mathrm{A}=\mathrm{R}=\mathrm{S}=\lambda \in\{0.1,0.2,0.5\}$ (respective means $10 \mathrm{~ms}, 5 \mathrm{~ms}$ and $2 \mathrm{~ms}$ ) across 50,000 writes. After empirically measuring the WARS distributions, consistency, and latency for each partial quorum configuration, we predicted $t$-visibility and latency. Our average $t$-visibility prediction RMSE was $0.28 \%$ (std. dev. $0.05 \%$, max. $0.53 \%$ ) for each $t \in\{1, \ldots, 199\}$ ms. Our predicted latency (for each of the $\{1.0, \ldots, 99.9$ th $\}$ percentiles for each configuration) had an average N-RMSE of $0.48 \%$ (std. dev. $0.18 \%$, max. 0.90\%). This validates our Monte Carlo simulator.

\subsection{Write Latency Distribution Effects}

As discussed in Section 4.1, the WARS model of Dynamo-style systems dictates that high one-way write variance $(\mathrm{W})$ increases staleness. To quantify these effects, we swept a range of exponentially distributed write distributions (changing parameter $\lambda$, which dictates the mean and tail of the distribution) while fixing $\mathrm{A}=\mathrm{R}=\mathrm{S}$.

Our results, shown in Figure 4, confirm that probability of consistency is highly influenced by the write variance. When the variance of $\mathrm{W}$ is $0.0625 \mathrm{~ms}$ ( $\lambda=4$, mean $.25 \mathrm{~ms}$, one-fourth the mean of $\mathrm{A}=\mathrm{R}=\mathrm{S}$ ), we observe a $94 \%$ chance of consistency immediately after the write and $99.9 \%$ chance after $1 \mathrm{~ms}$. However, when the variance of $\mathrm{W}$ is $100 \mathrm{~ms}(\lambda=.1$, mean $10 \mathrm{~ms}$, ten times the mean of $A=R=S$ ), we observe a $41 \%$ chance of consistency immediately after write and a $99.9 \%$ chance of consistency only after $65 \mathrm{~ms}$. As the variance and mean increase, so does the probability of inconsistency. Under distributions with fixed means and variable variances

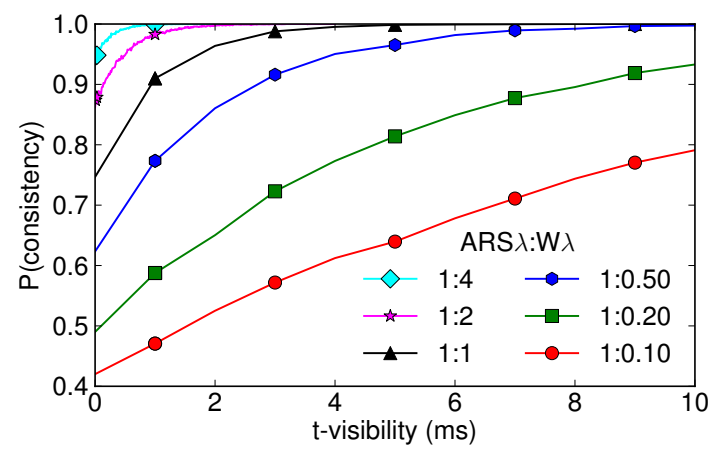

Figure 4: $t$-visibility for $N=3, R=W=1$ varying exponentially distributed latencies (parameter $\lambda$ ) for $\mathrm{W}$ and $\mathrm{A}=\mathrm{R}=\mathrm{S}$. Mean latency is $1 / \lambda$.

\begin{tabular}{|c|c|}
\hline$\%$ ile & Latency (ms) \\
\hline 15,000 RPM SAS Disk \\
\hline Average & 4.85 \\
95 & 15 \\
99 & 25 \\
\hline \multicolumn{2}{|c|}{ Commodity SSD } \\
\hline Average & 0.58 \\
95 & 1 \\
99 & 2 \\
\hline
\end{tabular}

Table 1: LinkedIn Voldemort single-node production latencies.

(uniform, normal), we observe that the mean of $\mathrm{W}$ is less important than its variance if $W$ is strictly greater than $A=R=S$.

Decreasing the mean and variance of $\mathrm{W}$ improves the probability of consistent reads. This means that, as we will see, techniques that lower one-way write latency result in lower $t$-visibility. Instead of increasing read and write quorum sizes, operators could chose to lower (relative) $\mathrm{W}$ latencies through additional hardware configuration and specialization or by artificially delaying reads. This latter option is potentially detrimental to performance for read-heavy workloads and may introduce undesirable queuing effects.

\subsection{Production Latency Distributions}

To study WARS in greater detail, rather than conjecture as to what represent "reasonable" distributions, we gathered production statistics from two internet-scale companies.

LinkedIn ${ }^{6}$ is an online professional social network with over 135 million members as of November 2011. To provide highly available, low latency data storage, engineers at LinkedIn built Voldemort. Alex Feinberg, a lead engineer on Voldemort, graciously provided us with latency distributions for a single node replaying peak load traffic for a user-facing service at LinkedIn, representing $60 \%$ read and $40 \%$ read-modify-write traffic [23] (Table 1). Feinberg reports that, using spinning disks, Voldemort is "largely IO bound and latency is largely determined by the kind of disks we're using, [the] data to memory ratio and request distribution." With solid state drives (SSDs), Voldemort is "CPU and/or network bound (depending on value size)." As an interesting aside, Feinberg also notes that "maximum latency is generally determined by [garbage collection] activity (rare, but happens occasionally) and is within hundreds of milliseconds."

Yammer $^{7}$ is an online, private social networking to over 100,000 companies as of December 2011 that uses Basho's Riak for some client data [3]. Coda Hale, infrastructure architect, and Ryan Kennedy,

\footnotetext{
${ }^{6}$ LinkedIn. www. linkedin. com

${ }^{7}$ Yammer. www. yammer. com
} 


\begin{tabular}{|c|c|c|}
\hline \%ile & Read Latency $(\mathrm{ms})$ & Write Latency $(\mathrm{ms})$ \\
\hline Min & 1.55 & 1.68 \\
50 & 3.75 & 5.73 \\
75 & 4.17 & 6.50 \\
95 & 5.2 & 8.48 \\
98 & 6.045 & 10.36 \\
99 & 6.59 & 131.73 \\
99.9 & 32.89 & 435.83 \\
Max & 2979.85 & 4465.28 \\
\hline Mean & 9.23 & 8.62 \\
Std. Dev. & 83.93 & 26.10 \\
\hline Mean Rate & 718.18 gets/s & $45.65 \mathrm{puts} / \mathrm{s}$ \\
\hline
\end{tabular}

Table 2: Yammer Riak $N=3, R=2, W=2$ production latencies.

\begin{tabular}{|c|c|}
\hline LNKD-SSD & $\begin{array}{l}\mathrm{W}=\mathrm{A}=\mathrm{R}=\mathrm{S}: \\
91.22 \%: \text { Pareto, } x_{m}=.235, \alpha=10 \\
\text { 8.78\%: Exponential, } \lambda=1.66 \\
\text { N-RMSE: } .55 \%\end{array}$ \\
\hline & $\mathrm{A}=\mathrm{R}=\mathrm{S}: \mathrm{LNKD}-\mathrm{SSD}$ \\
\hline LNKD-DISK & $\begin{array}{l}\text { W: } \\
\begin{array}{r}\text { 38\%: Pareto, } x_{m}=1.05, \alpha=1.51 \\
62 \%: \text { Exponential, } \lambda=.183 \\
\text { N-RMSE: } .26 \%\end{array}\end{array}$ \\
\hline \multirow{2}{*}{ YMMR } & $\begin{array}{r}\text { W: } \\
\text { 93.9\%: Pareto, } x_{m}=3, \alpha=3.35 \\
6.1 \%: \text { Exponential, } \lambda=.0028 \\
\text { N-RMSE: } 1.84 \%\end{array}$ \\
\hline & $\begin{array}{r}\mathrm{A}=\mathrm{R}=\mathrm{S}: \\
98.2 \%: \text { Pareto, } x_{m}=1.5, \alpha=3.8 \\
1.8 \%: \text { Exponential, } \lambda=.0217 \\
\text { N-RMSE: } .06 \%\end{array}$ \\
\hline
\end{tabular}

Table 3: Distribution fits for production latency distributions (see Appendix B) from LinkedIn (LNKD-*) and Yammer (YMMR).

also of Yammer, presented in-depth performance and configuration details for their Riak usage in March 2011 [31]. Hale provided us with more detailed performance statistics for their application [30] (Table 2). He also noted that "reads and writes have radically different expected latencies, especially for Riak." Writes are delayed "until the fsync returns, so while reads are often $<1 \mathrm{~ms}$, writes rarely are." As we will see, this has important consequences for WARS. Although we do not model this explicitly, Hale also notes that the size of values is important, claiming "a big performance improvement by adding LZF compression to values."

We fitted the distributions given by LinkedIn (LNKD-DISK and LNKD-SSD for the disk and SSD numbers, respectively) and Yammer (YMMR), as described in detail in Appendix B. We also considered a wide-area network replication scenario, denoted WAN. Reads and writes are routed to random data centers, and, accordingly, one replica operation completes quickly while the others are routed remotely. We delay remote operations and responses by $75 \mathrm{~ms}$ and apply LNKD-DISK delays once the operation reaches its target data center, representing multi-continent WAN network delay [20]

We show the parameters for each distribution in Table 3 . We plot each fitted distribution in Figure 5. Note that for $R, W$ of one, LNKD-DISK is not equivalent to WAN. This is because, in LNKD-DISK, we only have to wait for the first of $N$ local reads (writes) to return, whereas, for WAN, there is only one local read (write) and all other read (write) requests are delayed at least $150 \mathrm{~ms}$.

\subsection{Observed $t$-visibility}

We measured the $t$-visibility for each distribution (Figure 6). As we observed under synthetic distributions in Section 5.3, the $t$-visibility depended on both the relative mean and variance of $\mathrm{W}$.

LNKD-SSD and LNKD-DISK demonstrate the importance of write latency in practice. Immediately after write commit, LNKD-SSD had a $97.4 \%$ probability of consistent reads, reaching over a $99.999 \%$ probability of consistent reads after five milliseconds. LNKD-SSD's reads briefly raced its writes immediately after commit. Several millisecond after the write, the chance of a read round-trip experiencing reordering with the write was almost completely eliminated. The distribution's read and write operation latencies were rather small (median $.489 \mathrm{~ms}$ ), and due to its short tail (99.9th percentile $.657 \mathrm{~ms}$ ), writes completed quickly across all replicas. In contrast, under LNKD-DISK, writes take significantly longer (median $1.50 \mathrm{~ms}$ ) and have a longer tail (99.9th percentile $10.47 \mathrm{~ms}$ ). This difference is reflected in its $t$-visibility: LNKD-DISK had only a $43.9 \%$ probability of consistent reads immediately after write commit and only a $92.5 \%$ probability ten milliseconds later. This suggests that SSDs may drastically improve consistency in practice due to reducing replica write time.

We experienced similar effects with the other distributions. Immediately after commit, YMMR had a $89.3 \%$ chance of consistency due to its tall body. However, YMMR's long tail hampered its $t$ visibility increase and reached a $99.9 \%$ probability of consistency $1364 \mathrm{~ms}$ after commit. As expected, WAN observed poor chances of consistency until after the 75 milliseconds passed (33\% after commit); unless a client read from the same datacenter in which the last write committed, it had to wait for the long propagation delay to observe the most recent value.

\subsection{Quorum Sizing}

In addition to $N=3$, we consider how varying the number of replicas $(\mathrm{N})$ affects $t$-visibility while maintaining $R=W=1$. The results, depicted in Figure 7, show that the probability of consistency immediately after write commit decreases as $N$ increases. With 2 replicas, LNKD-DISK has a $57.5 \%$ probability of consistent reads immediately after commit but only a $21.1 \%$ probability with 10 replicas. However, at high probabilities of consistency, the wait time required for increased replica sizes is surprisingly close. For LNKD-DISK, the $t$-visibility at $99.9 \%$ probability of consistency ranges from $45.3 \mathrm{~ms}$ for 2 replicas to $53.7 \mathrm{~ms}$ for 10 replicas.

These results imply that even if we choose to maintain a large number of replicas for availability or better performance, the $t$ visibility staleness will still converge relatively quickly for larger $t$. However, increasing $N$ will have a larger impact on the consistency immediately after writing. In practice, the relative write distribution tail length (describing replica convergence speed) will again dictate whether this trade-off is appropriate.

\subsection{Latency vs. $t$-visibility}

As we have discussed, choosing a value for $R$ and $W$ is a tradeoff between operation latency and $t$-visibility. To measure the obtainable latency gains, we compared $t$-visibility required for a $99.9 \%$ probability of consistent reads to the 99.9th percentile read and write latencies.

Partial quorums can have a large impact on latency at a variable cost to $t$-visibility (Table 4). For YMMR, $R=W=1$ results in low latency reads and writes $(16.4 \mathrm{~ms})$ but high $t$-visibility $(1364 \mathrm{~ms})$. However, by setting $R=2$ and $W=1$, we reduce $t$-visibility to $202 \mathrm{~ms}$ and the combined read and write latencies are $81.1 \%(186.7 \mathrm{~ms})$ lower than the fastest strict quorum $(W=1, R=3$ ). LNKD-DISK read and write latencies can be reduced by $16.5 \%(2.48 \mathrm{~ms})$ with $t$-visibility of $13.6 \mathrm{~ms}$. The write tail of LNKD-SSD was such that we never observed message reordering for $R=2, W=1$, allowing a $30 \%(.98 \mathrm{~ms})$ latency reduction with no observable staleness (even 

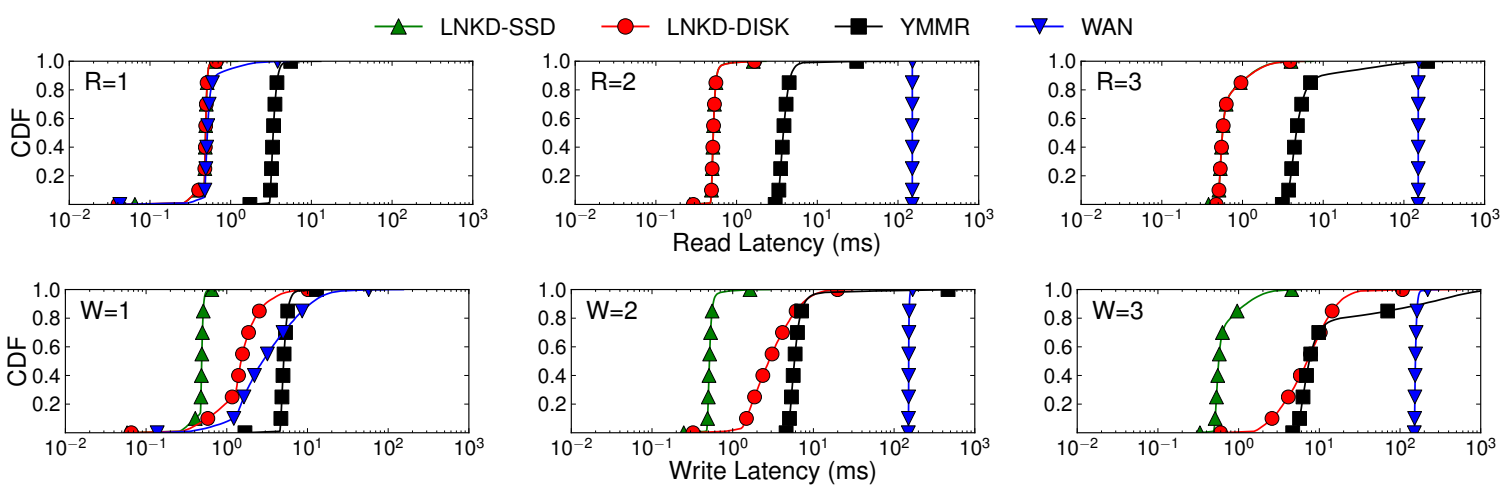

Figure 5: Read and write operation latency for production fits for $N=3$. Note that, for reads, LNKD-SSD is equivalent to LNKD-DISK.
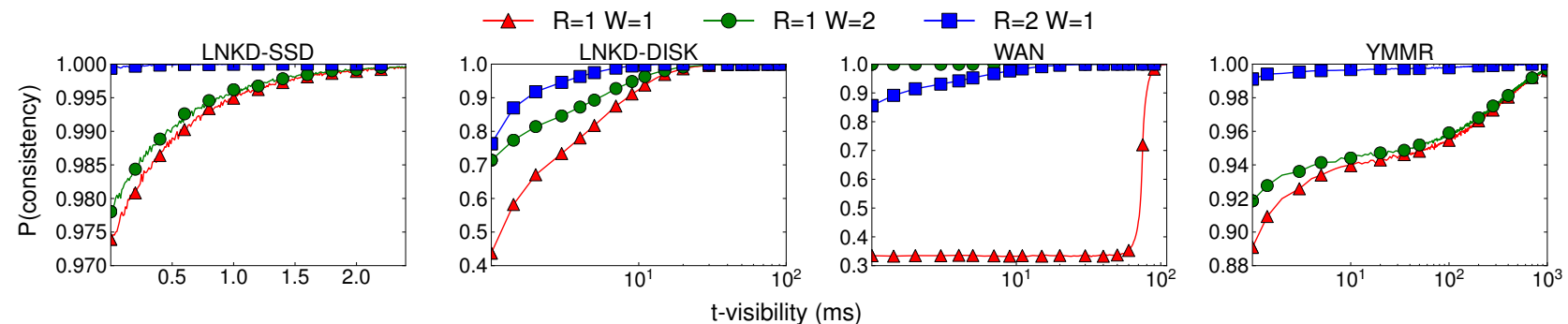

Figure 6: $t$-visibility for production operation latencies.

across $10 M$ writes, "seven nines"). $R=W=1$ reduced latency by $59.5 \%(1.94 \mathrm{~ms})$ with a corresponding $t$-visibility of $1.85 \mathrm{~ms}$. Under WAN, $R>1$ or $W>1$ results in a large latency increase because operations require remote messaging to complete. These results indicate that lowering values of $R$ and $W$ can significantly improve operation latency and that $t$-visibility can be low even when we require a high probability of consistent reads.

\section{DISCUSSION AND FUTURE WORK}

In this section, we discuss several enhancements to partial quorum systems that PBS enables along with future work for PBS.

Latency/Staleness SLAs. Using PBS, we can automatically configure replication parameters by optimizing operation latency given constraints on staleness and minimum durability. Data store operators can subsequently provide service level agreements to applications and quantitatively describe latency/staleness trade-offs to developers. Operators can dynamically configure replication using online latency measurements. PBS provides a quantitative lens for analyzing consistency guarantees that were previously unknown. This optimization formulation is likely non-convex, but the state space for configurations is small $\left(O\left(N^{2}\right)\right)$. This optimization also allows disentanglement of replication for reasons of durability from replication for reasons of low latency and higher capacity. For example, operators can specify a minimum replication factor for durability and availability but may want to automatically increase $N$, decreasing tail latency for fixed $R$ and $W$.

Variable configurations. We have assumed the use of a single replica configuration $(N, R$, and $W)$ across all operations. However, one could consider varying these operations over time and across keys, and many data stores such as Cassandra and Riak allow the use of per-operation consistency settings. By specifying an average operation latency, one could periodically modify $R$ and $W$ to more efficiently guarantee a desired bound on staleness. Similarly, varying load, one could change the the number of replicas and scale $R$ and $W$, requiring additional refinements to our model and essentially revisiting prior work on fluid replication [54].
Stronger guarantees. We have focused on probabilistic staleness analysis, but there is a range of stronger models (such as causal consistency) within the spectrum of eventual consistency [62]. Predicting the probability of attaining more complex consistency semantics requires additional modeling of application access patterns. This is possible, but we suspect that modeling the worst-case semantics of these operations will result in unfavorably low probabilities of consistent operations. We can see this in Aiyer et al.'s analysis of Byzantine $k$-quorums [11]: in a worst-case deployment, with an adversarial scheduler, the lower bound on staleness is quite high. We conjecture that the bound would be even higher had the authors performed an analysis of stronger consistency models.

Alternative architectures. Dynamo is conceptually easy to understand and implement (WARS) but is painful to analyze analytically. Is there a design that finds a better middle ground between operational elegance and simplicity of analysis within the eventually consistent design space? Prior work on deterministic bounded staleness (Section 7) provides guidance but often sacrifices availability and may be more complex to reason about.

Multi-key operations. We have considered single-key operations, however the ability to perform multi-key operations is potentially attractive. For read-only transactions, if the key distribution is random and each quorum is independent, we can multiply the staleness probabilities of each key. Achieving atomicity of writes to multiple keys requires more complicated coordination mechanisms such as two-phase commit, increasing operation latency. Transactions are feasible but require considerable care in implementation, complicating what is otherwise a simple replication scheme.

Failure modes. In our evaluation of $t$-visibility, we focused on normal operating conditions. Unless failures are common-case, they affect tail staleness probabilities (which appear as latency spikes in WARS). For example, if, as Jeff Dean of Google suggests [20], servers crash at least twice per year, given a ten hour downtime per failure, this roughly represents $.23 \%$ downtime per machine per year. If failures are correlated, this small percentage may be a problem. However, if they are independent, a replica set of $N$ nodes 

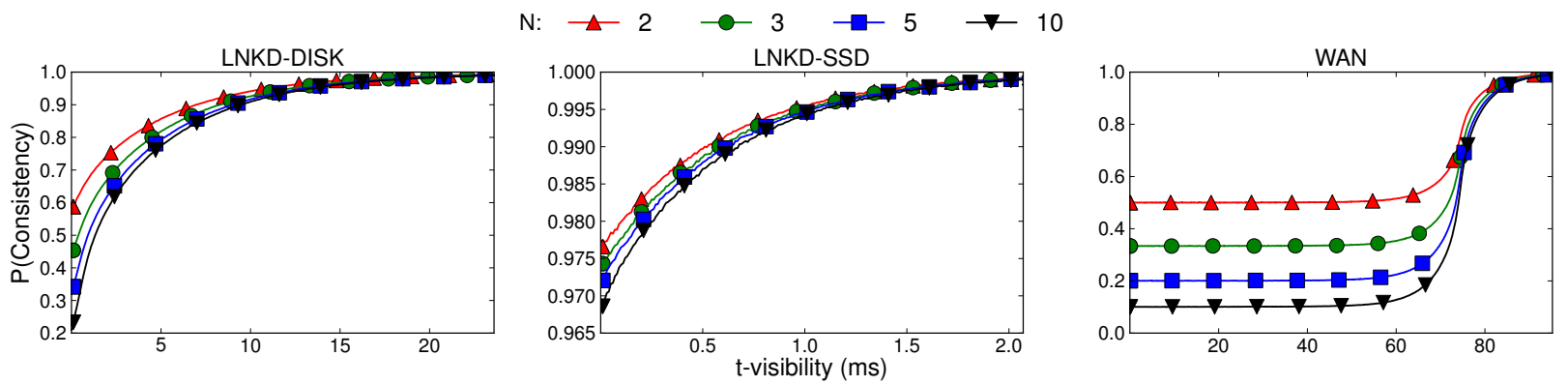

Figure 7: $t$-visibility for production operating latencies for variable $N$ and $R=W=1$.

\begin{tabular}{|c|c|c|c|c|c|c|c|c|c|c|c|c|}
\hline & \multicolumn{3}{|c|}{ LNKD-SSD } & \multicolumn{3}{|c|}{ LNKD-DISK } & \multicolumn{3}{|c|}{ YMMR } & \multicolumn{3}{|c|}{ WAN } \\
\hline & $L_{r}$ & $L_{w}$ & $t$ & $L_{r}$ & $L_{w}$ & $t$ & $L_{r}$ & $L_{w}$ & $t$ & $L_{r}$ & $L_{w}$ & $t$ \\
\hline$R=1, W=1$ & 0.66 & 0.66 & 1.85 & 0.66 & 10.99 & 45.5 & 5.58 & 10.83 & 1364.0 & 3.4 & 55.12 & 113.0 \\
\hline$R=1, W=2$ & 0.66 & 1.63 & 1.79 & 0.65 & 20.97 & 43.3 & 5.61 & 427.12 & 1352.0 & 3.4 & 167.64 & 0 \\
\hline$R=2, W=1$ & 1.63 & 0.65 & 0 & 1.63 & 10.9 & 13.6 & 32.6 & 10.73 & 202.0 & 151.3 & 56.36 & 30.2 \\
\hline$R=2, W=2$ & 1.62 & 1.64 & 0 & 1.64 & 20.96 & 0 & 33.18 & 428.11 & 0 & 151.31 & 167.72 & 0 \\
\hline$R=3, W=1$ & 4.14 & 0.65 & 0 & 4.12 & 10.89 & 0 & 219.27 & 10.79 & 0 & 153.86 & 55.19 & 0 \\
\hline$R=1, W=3$ & 0.65 & 4.09 & 0 & 0.65 & 112.65 & 0 & 5.63 & 1870.86 & 0 & 3.44 & 241.55 & 0 \\
\hline
\end{tabular}

Table 4: $t$-visibility for $p_{s t}=.001\left(99.9 \%\right.$ probability of consistency for 50,000 reads and writes) and 99.9 th percentile read $\left(L_{r}\right)$ and write latencies $\left(L_{w}\right)$ across $R$ and $W, N=3$ (1M reads and writes). Significant latency-staleness trade-offs are in bold.

with $F$ failed nodes behaves like an $N-F$ replica set. The probability of all $N$ nodes failing is $(.23)^{N} \%$ ("five nines" reliability for $N=3$ ) and will likely be hidden in the probability tail. Quantifying this impact more precisely would be beneficial but requires additional information about failure rates and their impact on latency distributions. Additionally, modeling recovery semantics such as hinted handoff would also be useful.

\section{RELATED WORK}

We surveyed quorum replication techniques $[7,8,9,10,11,26$, $29,34,37,50,52,53$ ] in Section 2. In this work, we specifically draw inspiration from probabilistic quorums [50] and deterministic $k$-quorums $[10,11]$ in analyzing expanding quorum systems and their consistency. We believe that revisiting probabilistic quorum systems - including non-majority quorum systems such as tree quorums - in the context of write propagation, anti-entropy, and Dynamo is a promising area for theoretical work.

Data consistency is a long-studied problem in distributed systems [19, 28] and concurrent programming [35]. Given the CAP Theorem and the inability to maintain all three of consistency, availability, and partition tolerance [27], data systems have turned to "eventually consistent" semantics to provide availability in the face of partitions [18, 62]. Real-time consistency (RTC) is the strongest consistency model achievable in an available, one-way convergent (eventually consistent) system [49], however there is a plethora of alternative consistency models offering various performance tradeoffs, from session guarantees [61] to causal+ consistency [47] and parallel snapshot isolation [58]. Instead of proposing a new consistency model and building a system implementing new semantics, we have examined what consistency existing, widely deployed quorum-replicated systems actually provide.

There are several systems that provide deterministic bounds on staleness. FRACS [67] allows replicas to buffer updates up to a given staleness threshold under several replication schemes, including master-drive and group gossip. AQuA [40] asynchronously propagates updates from a designated master to replicas that in turn serve reads with bounded staleness. AQuA actively selects which replicas to contact depending on response time predictions and a guaranteed staleness bound. TRAPP [55] provides trade-offs between precision and performance for continuously evolving numerical data. TACT $[65,66]$ models consistency along three axes: numerical error, order error, and staleness. TACT bounds staleness by ensuring that each replica (transitively) contacts all other replicas in the system within a given time window. Finally, POL [13] bounds the number of operations performed per query, trading operation latency at scale with the amount of data a particular query can access, impacting accuracy.

In this work, we analyze quorum replication systems and the consistency provided by real-world Dynamo-style quorum systems. The aforementioned deterministic bounded staleness systems represent the deterministic dual of PBS, and their algorithms could be employed in an expanding partial quorum system like Dynamo.

\section{CONCLUSION}

In this paper, we introduced Probabilistic Bounded Staleness, which models the expected staleness of data returned by eventually consistent quorum-replicated data stores. By extending prior theory on probabilistic quorum systems, we derived an analytical solution for the $k$-staleness of a partial quorum system, representing the expected staleness of a read operation in terms of versions. We analyzed the $t$-visibility, or expected staleness of a read in terms of real time, under Dynamo-style quorum replication. To do so, we developed the the WARS latency model to explain how message reordering leads to staleness under Dynamo. To examine the effect of latency on $t$-staleness in practice, we used real-world traces from internet companies to drive a Monte Carlo analysis. We find that eventually consistent quorum configurations are frequently consistent after tens of milliseconds while offering latency benefits, explaining the prevalence of partial quorum configurations in practice. We conclude that "eventually consistent" partial quorum replication schemes frequently deliver consistent semantics in practice due largely to the resilience of Dynamo-style messaging.

\section{Interactive Demonstration}

An interactive demonstration of Dynamo-style PBS is available online at http://www . bailis.org/projects/pbs/. 


\section{Acknowledgments}

The authors would like to thank Alex Feinberg and Coda Hale for their cooperation in providing real-world distributions for experiments and for exemplifying positive industrial-academic relations through their conduct and feedback.

The authors would also like to thank the following individuals whose discussions and feedback improved this work: Marcos Aguilera, Peter Alvaro, Eric Brewer, Neil Conway, Greg Durrett, Hariyadi Gunawi, Sam Madden, Bill Marczak, Kay Ousterhout, Christopher Ré, Scott Shenker, Sriram Srinivasan, Doug Terry, Greg Valiant, and Patrick Wendell. We would especially like to thank Bryan Kate for his extensive feedback and Ali Ghodsi, who, in addition to providing feedback, originally piqued our interest in theoretical quorum systems.

This work was supported in part by gifts from Google, SAP, Amazon Web Services, Blue Goji, Cloudera, Ericsson, General Electric, Hewlett Packard, Huawei, IBM, Intel, MarkLogic, Microsoft, NEC Labs, NetApp, Oracle, Quanta, Splunk, and VMware and by DARPA (contract \#FA8650-11-C-7136). This material is based upon work supported by the National Science Foundation Graduate Research Fellowship under Grant DGE 1106400.

\section{REFERENCES}

[1] Apache Cassandra 1.0 documentation: About data consistency in Cassandra. http: //www.datastax.com/docs/1.0/dml/data_consistency.

[2] Apache Cassandra Jira: "support session (read-after-write) consistency".

https://issues . apache.org/jira/browse/CASSANDRA-876. October 2010 (accessed 13 December 2011).

[3] Basho Riak. http://basho.com/products/riak-overview/.

[4] Cassandra 1.0 Thrift Configuration. https://github.com/apache/cassandra/blob/ cassandra-1.0/interface/cassandra.thrift.

[5] Cassandra wiki: Operations. http://wiki.apache.org/cassandra/Operations\# Repairing_missing_or_inconsistent_data. Accessed 13 December 2011.

[6] D. Abadi. DBMS Musings: Replication and the latency-consistency tradeoff. http://dbmsmusings.blogspot.com/2011/12/ replication-and-latency-consistency.html. 7 December 2011.

[7] I. Abraham and D. Malkhi. Probabilistic quorums for dynamic systems (extended abstract). In DISC 2003.

[8] D. Agrawal and A. E. Abbadi. Resilient logical structures for efficient management of replicated data. In $V L D B 1992$.

[9] D. Agrawal and A. E. Abbadi. The tree quorum protocol: An efficient approach for managing replicated data. In VLDB 1990.

[10] A. Aiyer, L. Alvisi, and R. A. Bazzi. On the availability of non-strict quorum systems. In DISC 2005.

[11] A. S. Aiyer, L. Alvisi, and R. A. Bazzi. Byzantine and multi-writer k-quorums. In DISC 2006.

[12] P. Alvaro, N. Conway, J. M. Hellerstein, and W. R. Marczak. Consistency Analysis in Bloom: a CALM and Collected Approach. In CIDR 2011.

[13] M. Armbrust, K. Curtis, T. Kraska, A. Fox, M. J. Franklin, and D. A. Patterson. PIQL: Success-tolerant query processing in the cloud. In VLDB 2012.

[14] Basho Technologies, Inc. Riak wiki: Riak $>$ concepts $>$ replication. http://wiki.basho.com/Replication.html. Accessed 13 December 2011.

[15] Basho Technologies, Inc. riak_kv 1.0 application. https://github.com/basho/riak_kv/blob/1.0/src/riak_ kv_app.erl.

[16] J. Bellis. Revision 986783: revert 'per-connection read-your-writes "session" consistency'. http://svn. apache.org/viewvc?view= revision\&revision=986783. 18 August 2010, one week after original patch accepted.

[17] K. Birman, G. Chockler, and R. van Renesse. Toward a cloud computing research agenda. SIGACT News, 40(2):68-80, June 2009.

[18] S. Davidson, H. Garcia-Moina, and D. Skeen. Consistency in partitioned networks. ACM Computing Surveys, 17(3):314-370, 1985.

[19] S. B. Davidson. Optimism and consistency in partitioned distributed database systems. ACM Transactions on Database Systems, 9(3):456-481, September 1984.

[20] J. Dean. Designs, lessons, and advice from building large distributed systems. Keynote from LADIS 2009.

[21] G. DeCandia, D. Hastorun, M. Jampani, G. Kakulapati, A. Lakshman, A. Pilchin, S. Sivasubramanian, P. Vosshall, and W. Vogels. Dynamo: Amazon's highly available key-value store. In SOSP 2007.

[22] A. Demers, D. Greene, C. Hauser, W. Irish, J. Larson, S. Shenker, H. Sturgis, D. Swinehart, and D. Terry. Epidemic algorithms for replicated database maintenance. In PODC 1987.

[23] A. Feinberg. Personal communication. 23, 24 October, 14, 19, 21, 30 November, 1 December 2011.

[24] A. Feinberg. Project Voldemort: Reliable distributed storage. In ICDE 2011.

[25] A. W. Fu. Delay-optimal quorum consensus for distributed systems. IEEE Transactions on Parallel and Distributed Systems, 8(1):59-69, 1997.

[26] D. K. Gifford. Weighted voting for replicated data. In SOSP 1979.

[27] S. Gilbert and N. Lynch. Brewer's conjecture and the feasibility of consistent, available, partition-tolerant web services. SIGACT News, 33:51-59, June 2002.

[28] J. Gray, P. Helland, P. ONeil, and D. Shasha. The dangers of replication and a solution. In SIGMOD 1996.

[29] A. Gupta, B. M. Maggs, F. Oprea, and M. K. Reiter. Quorum placement in networks to minimize access delays. In PODC 2005.

[30] C. Hale. Personal communication. 16 November 2011.

[31] C. Hale and R. Kennedy. Using Riak at Yammer. http://dl. dropbox.com/u/2744222/2011-03-22_Riak-At-Yammer.pdf. 23 March 2011.

[32] J. Hamilton. Perspectives: I love eventual consistency but... http://perspectives.mvdirona.com/2010/02/24/ ILoveEventualConsistencyBut . aspx. 24 February 2010.

[33] P. Helland and D. Campbell. Building on quicksand. In CIDR 2009.

[34] M. Herlihy. Dynamic quorum adjustment for partitioned data. ACM Transactions on Database Systems, 12 (2):170-194, 1987.

[35] M. Herlihy and J. M. Wing. Linearizability: a correctness condition for concurrent objects. ACM Transactions on Programming Languages and Systems, 12(3):463-492, June 1990.

[36] P. Hunt, M. Konar, F. P. Junqueira, and B. Reed. ZooKeeper: Wait-free coordination for internet-scale systems. In USENIX ATC 2010.

[37] R. Jiménez-Peris, M. Patiño Martínez, G. Alonso, and K. Bettina. Are quorums an alternative for data replication? ACM Trans. Database Syst., 28:257-294, September 2003.

[38] D. King. As keltranis, comment on "reddit's now running on cassandra". http://www.reddit.com/r/programming/comments/bcqhi/ reddits_now_running_on_cassandra/c0m3wh6. March 2010.

[39] J. Kirkell. Consistency or bust: Breaking a Riak cluster. http://www.oscon.com/oscon2011/public/schedule/ detail/19762. Talk at O'Reilly OSCON 2011, 27 July 2011.

[40] S. Krishnamurthy, W. H. Sanders, and M. Cukier. An adaptive quality of service aware middleware for replicated services. IEEE Transactions on Parallel and Distributed Systems, 14(11), November 2003.

[41] A. Lakshman, P. Malik, and K. Ranganathan. Cassandra: A structured storage system on a P2P network. In SIGMOD 2008.

[42] L. Lamport. Time, clocks, and the ordering of events in a distributed system. Communications of the ACM, 21(7):558-565, 1978.

[43] L. Lamport. The part-time parliament. ACM Transactions on Computer Systems, 16(2):133-169, 1998.

[44] G. Linden. Make data useful. https://sites.google.com/ 
site/glinden/Home/Stanf ordDataMining . 2006-11-29.ppt. 29 November 2006.

[45] G. Linden. Marissa Mayer at Web 2.0. http://glinden. blogspot.com/2006/11/marissa-mayer-at-web-20.html. 9 November 2006.

[46] B. Liskov. Practical uses of synchronized clocks in distributed systems. In PODC 1991.

[47] W. Lloyd, M. J. Freedmand, M. Kaminsky, and D. G. Andersen. Don't settle for eventual: Scalable causal consistency for wide-area storage with COPS. In SOSP 2011.

[48] J. Lynch. Rolling with Riak. http://sdruby .org/podcast/81. Talk presented at SD Ruby meeting (Podcast 81), 2010.

[49] P. Mahajan, L. Alvisi, and M. Dahlin. Consistency, availability, convergence. Technical Report TR-11-22, Computer Science Department, University of Texas at Austin, May 2011.

[50] D. Malkhi, M. Reiter, A. Wool, and R. Wright. Probabilistic quorum systems. Information and Communication, (170):184-206, 2001.

[51] A. Marcus. The NoSQL Ecosystem. In A. Brown and G. Wilson, editors, The Architecture of Open Source Applications, pages 185-205. 2011.

[52] M. Merideth and M. Reiter. Selected results from the latest decade of quorum systems research. In Replication, volume 5959 of Lecture Notes in Computer Science, pages 185-206. Springer, 2010.

[53] M. Naor and A. Wool. The load, capacity, and availability of quorum systems. SIAM Journal on Computing, 27:214-225, 1998.

[54] B. Noble, B. Fleis, and M. Kim. A case for fluid replication. In Proceedings of the 1999 Network Storage Symposium (Netstore).

[55] C. Olston and J. Widom. Offering a precision-performance tradeoff for aggregation queries over replicated data. In VLDB 2000.

[56] Outbrain Inc. Introduction to no:sq1 [sic] and Cassandra (and Outbrain). https ://docs . google.com/present/view?id= ahbp3bktzpkc_220f7v26vg7. January 2010.

[57] E. Schurman and J. Brutlag. Performance related changes and their user impact. Presented at Velocity Web Performance and Operations Conference, June 2009.

[58] Y. Sovran, R. Power, M. K. Aguilera, and J. Li. Transactional storage for geo-replicated systems. In SOSP 2011.

[59] M. Stonebraker. Urban myths about SQL. VoltDB Webinar, 11 June 2010.

[60] R. Sumbaly. Voldemort wiki: How to write own client. https://github.com/voldemort/voldemort/wiki/ Writing-own-client-for-Voldemort. 16 June 2011 (accessed 21 December 2011).

[61] D. B. Terry, A. J. Demers, K. Petersen, M. J. Spreitzer, M. M. Theimer, and B. B. Welch. Session guarantees for weakly consistent replicated data. In PDIS 1994.

[62] W. Vogels. Eventually consistent. Commun. ACM, 52:40-44, January 2009.

[63] B. Wester, J. Cowling, E. B. Nightingale, P. M. Chen, J. Flinn, and B. Liskov. Tolerating latency in replicated state machines through client speculation. In NSDI 2009.

[64] D. Williams. HBase vs Cassandra: why we moved. http://ria101. wordpress.com/2010/02/24/ hbase-vs-cassandra-why-we-moved. 24 February 2010 (accessed 13 December 2011).

[65] H. Yu and A. Vahdat. Design and evaluation of a conit-based continuous consistency model for replicated services. ACM Transactions on Computer Systems, 20(3):239-282, August 2002.

[66] H. Yu and A. Vahdat. The costs and limits of availability for replicated services. ACM Transactions on Computer Systems, 24(1):70-113, February 2006.

[67] C. Zhang and Z. Zhang. Trading replication consistency for performance and availability: an adaptive approach. In ICDCS 2003.

\section{APPENDIX A: LOAD AND PBS $k$-STALENESS}

Theory literature defines the load of a quorum system as a metric for the frequency of accessing the busiest quorum member [53, Definition 3.2]. Intuitively, the busiest quorum member limits the number of requests that a given quorum system can sustain, called its capacity [53, Corollary 3.9].
Prior work determined that probabilistic quorum systems did not offer significant benefits to load (providing a constant factor improvement compared to strict quorum systems) [50]. Here, we show that quorums tolerating PBS $k$-staleness have asymptotically lower load than traditional probabilistic quorum systems (and, transitively, than strict quorum systems).

The probabilistic quorum literature defines an $\varepsilon$-intersecting quorum system as a quorum system that provides a $1-\varepsilon$ probability of returning consistent data [50, Definition 3.1]. A $\varepsilon$-intersecting quorum system has load of at least $\frac{1-\sqrt{\varepsilon}}{\sqrt{N}}$ [50, Corollary 3.12].

In considering $k$ versions of staleness, we consider the intersection of $k \varepsilon$-intersecting quorum systems. For a given probability $p$ of inconsistency, if we are willing to tolerate $k$ versions of staleness, we need only require that that $\varepsilon=\sqrt[k]{p}$. This implies that our PBS $k$-staleness system construction has load of at least $\frac{(1-p)^{\frac{1}{2 k}}}{\sqrt{N}}$, an improved lower bound compared to traditional probabilistic quorum systems. PBS monotonic reads consistency results in a lower bound on load of $\frac{(1-p)^{\frac{1}{2 C}}}{\sqrt{N}}$, where $C=1+\frac{\gamma_{g w}}{\gamma_{c r}}$.

These results are intuitive: if we are willing to tolerate multiple versions of staleness, we need to contact fewer replicas. Staleness tolerance lowers the load of a quorum system, subsequently increasing its capacity.

\section{APPENDIX B: LATENCY MODEL FITTING}

While the provided production figures are invaluable, they are underspecified for WARS. First, the data are summary statistics, but WARS requires distributions. More importantly, the operation latencies represent round-trip times, while WARS requires the constituent one-way latencies for both reads and writes. As our validation demonstrated, these latency distributions are easily collected, but, because they are not currently collected in production, we must fill in the gaps. Accordingly, to fit W, A, R, and S for each configuration, we made a series of assumptions, which we believe are justified given the benefit of production data. Without additional data on the latency required to read multiple replicas, we assume that each latency distribution is independently, identically distributed (IID). Each configuration fit a mixture model with two separate distributions, one for the body and the other for the tail.

LinkedIn provided two latency distributions, whose fits we denote LNKD-SSD and LNKD-DISK for the SSD and spinning disks, respectively. As previously discussed, when running on SSDs, Voldemort is largely network and CPU bound. Accordingly, for LNKD-SSD, we assumed that read and write operations took equivalent amounts of time and, to allocate the remaining time, we focused on the network-bound case and assumed that one-way messages were symmetric $(\mathrm{W}=\mathrm{A}=\mathrm{R}=\mathrm{S})$. Feinberg reported that Voldemort performs at least one read before every write (average of 1 seek, between 1-3 seeks), and writes to the BerkeleyDB Java Edition backend are flushed either every 30 seconds or 20 megabyteswhichever comes first. Accordingly, for LNKD-DISK, we used the same $A=R=S$ as LNKD-SSD but fit $W$ separately.

Yammer provided distributions for a single configuration, denoted YMMR, but separated read and write latencies. Under our IID assumptions, we fit single-node latency distributions to the provided data, again assuming symmetric $\mathrm{A}, \mathrm{R}$, and $\mathrm{S}$. The data again fit a Pareto distribution with a long exponential tail. At the 98th percentile, the write distribution takes a sharp turn. Fitting the data closely resulted in an extremely long tail, with 99.99+th percentile writes requiring tens of seconds-much higher than Yammer specified. Accordingly, we fit the 98th percentile knee conservatively; without the 98 th percentile, the write fit N-RMSE is .104\%. 